\title{
Materials research under ITER-like divertor conditions at FOM Rijnhuizen
}

\author{
G.M. Wright ${ }^{\mathrm{a}, \mathrm{b}, *}$, J. Westerhout $^{\mathrm{a}}$, R.S. Al ${ }^{\mathrm{a}}$, E. Alves ${ }^{c}$, L.C. Alves ${ }^{c}$, N.P. Barradas ${ }^{c}$, M.A. van den Berg ${ }^{\mathrm{a}}$, \\ D. Borodin ${ }^{\mathrm{d}}$, S. Brezinsek ${ }^{\mathrm{d}}$, S. Brons ${ }^{\mathrm{a}}$, H.J.N. van Eck ${ }^{\mathrm{a}}$, B. de Groot ${ }^{\mathrm{a}}$, A.W. Kleyn ${ }^{\mathrm{a}, \mathrm{e}}$, W.R. Koppers ${ }^{\mathrm{a}}$, \\ O.G. Kruijt ${ }^{a}$, J. Linke ${ }^{d}$, N.J. Lopes Cardozo ${ }^{f}$, M. Mayer ${ }^{\text {}}$, H.J. van der Meiden ${ }^{a}$, P.R. Prins ${ }^{a}$, G.J. van Rooij ${ }^{a}$, \\ J. Scholten ${ }^{a}$, A.E. Shumack ${ }^{\text {a }}$ P.H.M. Smeets ${ }^{a}$, G. De Temmerman ${ }^{\text {h }}$, W.A.J. Vijvers ${ }^{\text {a }}$, J. Rapp ${ }^{\text {a,d }}$ \\ ${ }^{a}$ FOM-Institute for Plasma Physics Rijnhuizen, Association EURATOM, A Member of the Trilateral Euregio Cluster, Postbus 1207,3430 BE, Nieuwegein, The Netherlands \\ ${ }^{\mathrm{b}}$ MIT Plasma Science and Fusion Center, 77 Massachusetts Ave. Cambridge, MA 02139, USA \\ ${ }^{\mathrm{c}}$ Instituto Tecnológico e Nuclear, EN. 10, 2686-953 Sacavém, Portugal \\ d Institut für Energie forschung-Plasmaphysik, Forschungszentrum Jülich, Association EURATOM, Trilateral Euregio Cluster, 52425 Jülich, Germany \\ e Leiden Institute for Chemistry, Leiden University, Postbus 9502, 2300 RA, Leiden, The Netherlands \\ ${ }^{\mathrm{f}}$ Eindhoven University of Technology, Postbus 513, 5600 MB, Eindhoven, The Netherlands \\ ${ }^{g}$ Max-Planck-Institut für Plasmaphysik, Association EURATOM, Postfach 1317, D-85741, Garching, Germany \\ ${ }^{\mathrm{h}}$ EURATOM/UKAEA Fusion Association, Culham Science Centre, Abingdon, OX14 3DB, UK
}

\section{A R T I C L E I N F O}

Article history:

Available online 25 December 2010

\begin{abstract}
A B S T R A C T
At FOM Rijnhuizen, linear plasma generators are used to investigate plasma-material interactions under high-density $\left(\leqslant 10^{21} \mathrm{~m}^{-3}\right)$, low-temperature $(\leqslant 5 \mathrm{eV})$ plasma bombardment. Research into carbon-based materials has been focused on chemical erosion by hydrogen plasmas. Results from plasma exposure to high-flux $\left(>10^{23} \mathrm{H}^{+} / \mathrm{m}^{2} \mathrm{~s}\right)$ and low-temperature hydrogen plasma indicate silicon carbide has a lower relative rate of gross erosion than other carbon-based materials (e.g. graphite, diamond, carbon-fiber composites) by a factor of 7-10. Hydrogenic retention is the focus of research on tungsten and molybdenum. For target temperatures of $700-1600 \mathrm{~K}$, the temperature dependence of hydrogenic retention is the dominant factor. Damage to the surface by heavy ion irradiation has shown to enhance retention by a factor of 2.5-4.1. Thermal stressing of $W$ via. e-beam thermal cycling also enhances hydrogenic retention by a factor of $2.1 \pm 0.2$, likely due to the introduction of thermal defects, which act as trapping sites for implanted hydrogenic isotopes.
\end{abstract}

(c) 2011 Elsevier B.V. All rights reserved.

\section{Introduction}

For next generation fusion devices such as ITER, plasma-surface interactions (PSI) is still an area of great uncertainty. One of the main obstacles is the difficulty in recreating the plasma conditions expected in the divertor region of a next generation fusion device. Current tokamaks cannot re-create the high plasma fluxes expected at the divertor strikepoints of future fusion devices including ITER. In addition, diagnostic and physical access to the plasma-facing materials in tokamak divertors is limited, making controlled studies of the evolution of materials difficult.

The goal of the PSI group at FOM Rijnhuizen is to re-create these plasma conditions in a laboratory setting with improved physical and diagnostic access to the plasma and the material being tested. Dedicated PSI experiments examine the effects of high-flux $\left(\geqslant 10^{23} \mathrm{~m}^{-2} \mathrm{~s}^{-1}\right)$, low electron temperature $\left(T_{e}<10 \mathrm{eV}\right)$ plasmas on

\footnotetext{
* Corresponding author at: PSFC MIT, 175 Albany St. NW17-121, Cambridge, MA 02139, USA. Tel.: +1 617253 5956; fax: +1 6172530627.

E-mail address: wright@psfc.mit.edu (G.M. Wright).
}

fusion relevant materials such as carbon-based materials and refractory metals. A new linear plasma device, MAGNUM-PSI [1], will begin regular plasma operations by the end of 2009. This device can operate in steady-state with the use of a $3 \mathrm{~T}$ super-conducting magnet, with a plasma column diameter of $100 \mathrm{~mm}$. Current research is performed on a smaller forerunner experiment, Pilot-PSI, which is limited to pulsed operation, a maximum magnetic field of $1.6 \mathrm{~T}$ and a narrow ( $\sim 20 \mathrm{~mm}$ ) column width. Nevertheless, both experiments are able to achieve high-density $\left(n_{e} \geqslant 10^{20} \mathrm{~m}^{-3}\right)$ low temperature $\left(T_{e}<5 \mathrm{eV}\right)$ plasma from a cascaded arc plasma source [2].

The work with the carbon-based materials mainly focuses on determining chemical erosion yields in this high-flux plasma regime. The chemical erosion of carbon-based materials has never been measured at the high fluxes, and low electron temperatures expected at the ITER divertor strikepoints. This represents a serious gap in our knowledge since the divertor strikepoints are region where carbon-based materials, specifically carbon-fiber composites (CFC), will be used in ITER. There are also a wide-range of proposed carbon-based materials for use in ITER and future fusion reactors and a relative comparison of gross carbon erosion for these different materials is presented in this work. 
For refractory metals, the focus of research has been on the hydrogenic retention of the material. Laboratory results have established that hydrogenic retention of the refractory metals tungsten (W) and molybdenum (Mo) is very low. However, these laboratory experiments do not take into consideration many factors that will play a role in future fusion devices such as high $\left(>10^{23} \mathrm{~m}^{-2} \mathrm{~s}^{-1}\right)$ ion fluxes, neutron irradiation and thermal stressing resulting in surface cracking. For example, there are concerns that high plasma fluxes to a material with low hydrogen solubility, such as tungsten or molybdenum, could lead to a build up of lateral stresses in the implantation zone, resulting in the formation of vacancies, dislocations or even voids and surface blistering [3-5]. The linear plasma generators at FOM Rijnhuizen allow us to examine the impact of high plasma fluxes on the hydrogenic retention of these low hydrogen solubility metals. Collaborations have also allowed us to examine retention in a high-flux plasma with thermally stressed tungsten (via. thermal cycling with an electron gun) and heavy ion-irradiated (a proxy for neutron irradiation) tungsten.

\section{Experimental}

\subsection{Material}

For the work with carbon, a wide range of carbon-based materials were used; fine- grain graphite (FGG), ITER-grade CFC (NB31, NB41 and Dunlop 3-D), silicon carbide (SiC), and nanocrystalline (NCD) and microcrystalline (MCD) diamond coatings. Targets were typically disks with $30 \mathrm{~mm}$ diameter and $4 \mathrm{~mm}$ thickness. The refractory metals, tungsten and molybdenum, have a minimum purity of $99.95 \%$ by weight and were polycrystalline. These were disks with $20 \mathrm{~mm}$ diameter and $1 \mathrm{~mm}$ thickness and were typically exposed in the "as received" condition (i.e. unpolished and unannealed). The reasoning behind exposing these targets in the "as received" condition was to best approximate the condition of the tungsten surfaces in a tokamak divertor during typical operations. It is already well-established that pre-annealing and polishing targets can affect retention properties, namely that pre-annealing of targets can reduce retention [6,7], while polishing can increase retention [8].

\subsection{Experimental methods}

All experiments were performed in the linear plasma device Pilot-PSI. For more details on Pilot-PSI we refer to [2]. The plasma electron density $\left(n_{e}\right)$ and temperatures $\left(T_{e}\right)$ are measured with Thomson scattering [9]. In Pilot-PSI, $n_{e}$ and $T_{e}$ follow a peaked distribution across the plasma column width leading to a peaked distribution in plasma flux density (see Fig. 1). Heat fluxes, and thus surface temperatures, are determined entirely by the plasma and thus also follow a peaked profile. Target temperatures are measured by a multi-wavelength spectropyrometer and/or a thermocouple inserted into the bulk of the material in combination with cooling water calorimetry to determine the total power to the target. Some investigations have also used a 2-D IR camera to determine temperature spatial profiles (see Fig. 1). Targets are mechanically attached to a water cooled heatsink and can either be electrically grounded, floating or actively biased. Due to limited magnet cooling, Pilot-PSI is a pulsed device. However, it should be noted that the plasma source is run in steady-state and exposures are initiated with the presence of the magnetic field. Thus the plasma source is well-conditioned before any targets are exposed to plasma. It should also be noted that time-resolved surface temperature measurements show that the target reaches approximate thermal equilibrium within the first

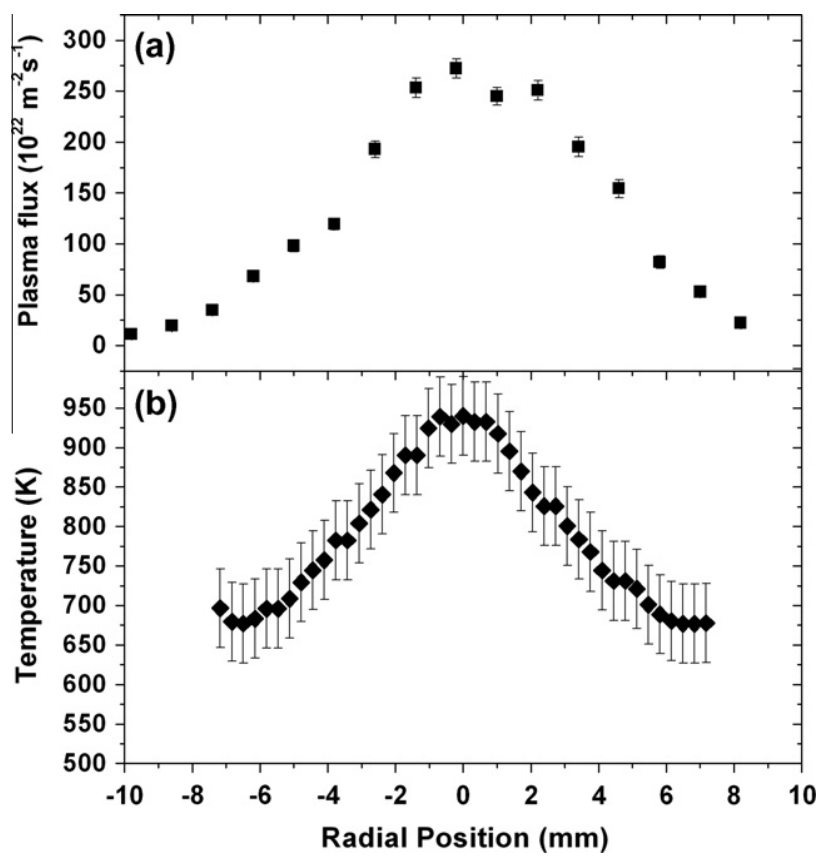

Fig. 1. (a) Typical radial distribution of incident plasma flux density in Pilot-PSI as determined from Bohm criterion calculations using electron density $\left(n_{e}\right)$ and temperature $\left(T_{e}\right)$ profiles from Thomson scattering data. (b) Surface temperature profile for a $\mathrm{W}$ target as measured by 2-D IR camera and central temperature confirmed with multi-wavelength pyrometer. Note that surface temperatures for carbon targets can be quite different since this is largely determined by the thermal contact of the re-deposited carbon on the surface.

$1-2 \mathrm{~s}$ of the plasma exposure. Pulse lengths are determined by magnetic field strength, ranging from $120 \mathrm{~s}(B=0.4 \mathrm{~T})$ to $4 \mathrm{~s}(B=1.6 \mathrm{~T})$.

For carbon erosion studies, chemical erosion is measured with optical emission spectroscopy with a high-resolution $(\sim 0.1 \mathrm{~nm}$ FWHM) spectrometer. The erosion is directly related to the total photon flux from the excitation band of the $\mathrm{CH}$ molecule (430.0$431.5 \mathrm{~nm}$ ), meaning a comparison of $\mathrm{CH}$ photon fluxes is a valid method for comparing erosion yields of different carbon-based materials. A consequence of this method is that it only measures gross erosion rather than net erosion. The advantages are that it is an in situ and non-invasive diagnostic, it can be spatially resolved with the proper optics system, and is performed during the plasma exposure rather than depending on post-mortem analysis.

For tungsten and molybdenum studies, hydrogenic retention is measured by nuclear reaction analysis (NRA) and thermal desorption spectroscopy (TDS). The NRA exploits the $\mathrm{d}\left({ }^{3} \mathrm{He}, \mathrm{p}\right) \alpha$ reaction to determine concentrations and depth profiles of the deuterium content in the near-surface $(<5 \mu \mathrm{m})$ region. NRA also allows for analysis of local concentrations giving a 2-D mapping of the retention across the surface of the target. For TDS, the target is linearly ramped to a temperature of $1273 \mathrm{~K}$ at a rate of $\sim 1 \mathrm{~K} / \mathrm{s}$ while a quadrupole mass spectrometer (QMS) monitors the mass $4\left(D_{2}\right)$ and mass 3 (HD) signals in the residual gas. These QMS signals are quantified into desorption rates using calibrated leaks of $D_{2}$ and $\mathrm{H}_{2}$.

\section{Results and discussion}

\subsection{Chemical erosion of carbon-based materials}

One of the key open questions of chemical erosion is the yield dependence on $T_{e}$. It is not clear how quickly carbon will chemically erode in the high-density, low-temperature plasma that is 
expected in a detached ITER divertor. With the Pilot-PSI experiment, the gross erosion yields under these conditions can be measured directly. Carbon-based targets are exposed to high-density $\left(\sim 10^{20} \mathrm{~m}^{-3}\right)$, low temperatures $(0.1-2.5 \mathrm{eV})$ plasmas for short discharges [10]. Hydrogen ion $\left(\mathrm{H}^{+}\right)$flux densities were on the order of $10^{24} \mathrm{~m}^{-2} \mathrm{~s}^{-1}$.

Gross erosion is known to be related to the detected photon flux from the $\mathrm{CH}$ excitation band through inverse photon efficiencies $(\mathrm{D} / \mathrm{XB})$ [11]. Measured $\mathrm{CH}$ photon fluxes appear constant over the temperature range of $1.0-2.5 \mathrm{eV}$, but decrease rapidly when $T_{e}$ is lower than $1.0 \mathrm{eV}$ (see Fig. 2). It is not clear if this dependence on $T_{e}$ is an effect of carbon recycling (i.e. erosion of re-deposited layers), changing plasma flux densities (i.e. plasma detachment) or is an erosion property for carbon. This is a topic for future work, but all materials tested follow this general $T_{e}$ dependence. It is important to note that all different materials, whether it be graphite (FGG), carbon-fiber composites (NB31, NB41, Dunlop 3-D) or diamond films (NCD, MCD), exposed to these conditions have very similar erosion rates with the exception of the $\mathrm{SiC}$. SiC has roughly an order of magnitude lower $\mathrm{CH}$ photon flux than other carbonbased materials, indicating a lower gross chemical erosion yield in comparison. The FGG has approximately twice the gross erosion rate at $T_{e}<1 \mathrm{eV}$ as compared to other species (e.g. NB31, NB41, Dunlop 3-D, NCD, MCD). For $T_{e}>1 \mathrm{eV}$, the erosion rate for all pure carbon materials (i.e. all materials excluding $\mathrm{SiC}$ ), are identical within the error bars of the measurement.

The significantly lower $\mathrm{CH}$ photon flux, and thus gross erosion rate, from the $\mathrm{SiC}$ is of particular interest. It is expected that the $\mathrm{CH}$ photon flux will be lower from $\mathrm{SiC}$ since it has $50 \%$ less carbon content than the graphite, CFC, or diamond targets. However, this should only account for a factor of 2 difference if all else is equal. The results in Fig. 2 show that $\mathrm{SiC}$ has a $\mathrm{CH}$ photon flux a factor of 7-10 less than the other, pure carbon materials investigated. A surface temperature dependence may be playing a role here since chemical erosion at low ion energies is known to depend on surface temperature [12,13] showing chemical erosion yields to decrease at high temperatures. It has also been demonstrated that the thermal conductivity of SiC can be substantially degraded under high thermal loading [14], which could lead to a higher surface temperature for the SiC targets. However, multi-wavelength pyrometry measurements of the surface temperature of the $\mathrm{SiC}$ during exposure (1100-1250 K) are in-line with pyrometry measurements of the graphite targets (1200-1300 K). In addition, CFC and diamond also have much different thermal conductivity than graphite and yet their $\mathrm{CH}$ photon fluxes are quite similar. Literature shows that total ( $\mathrm{Si}$ and $\mathrm{C}$ ) gross erosion of $\mathrm{SiC}$ is a factor of 4-5

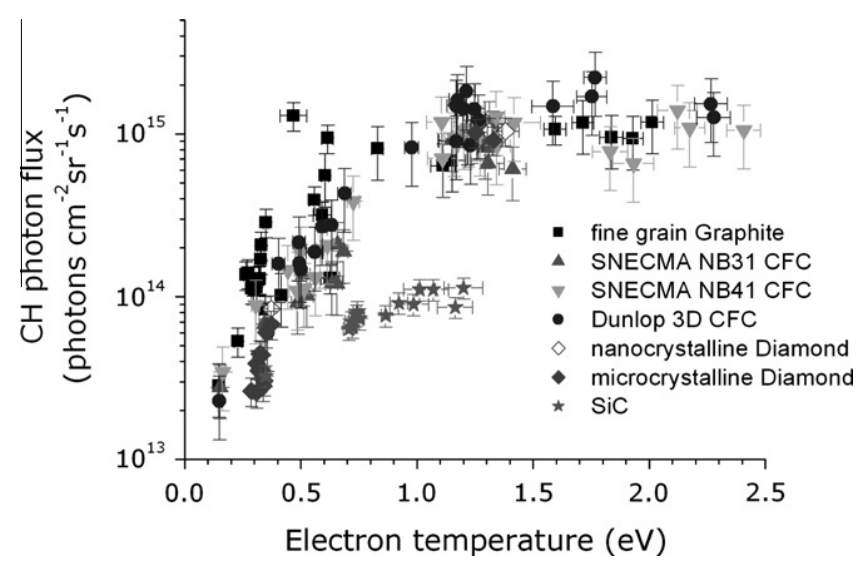

Fig. 2. $\mathrm{CH}$ photon flux as a function of plasma electron temperature in the range $0.1-2.5 \mathrm{eV}$ for a range of carbon-based materials. lower than for pure carbon at low ion energies $(20 \mathrm{eV})$ for temperatures up to $1100 \mathrm{~K}$ [15], which, when taking into account the factor of 2 lowering of $\mathrm{CH}$ photon flux from $\mathrm{Si}$ content in the $\mathrm{SiC}$, is in good agreement with our spectroscopic measurements. So it would appear that the reduced gross erosion rate seen for SiC can be attributed to a physical property of the material rather than changing experimental conditions for these exposures. This reduced gross erosion yield strengthens the case for using $\mathrm{SiC}$ as divertor plasma-facing components in future fusion devices.

The erosion and deposition of carbon also significantly changes the surface structure and roughness of the target. In Fig. 3, scanning electron microscopy (SEM) images are taken from unexposed, eroded and deposited surfaces of a graphite target. From the images it is clear that both erosion and deposition increase the surface roughness of the target. The nature of the surface roughness is different for erosion and deposition regions. The erosion region have more peaked structures. These can also be seen in profilometry measurements of erosion "craters". The deposition regions seem to have more clustered growth, with rounded structures on the surface. This could indicate seed regions for deposition and the structures grow out from these points or that the deposition is mostly from macroscopic particles or clusters of carbon that then adhere to the target surface rather than a purely atomic deposition process.

\subsection{Hydrogenic retention in tungsten and molybdenum}

To investigate the hydrogenic retention behaviour of refractory metals under high-flux plasma bombardment, $\mathrm{W}$ and Mo targets are exposed to high-density $\left(n_{e, \text { center }}>10^{20} \mathrm{~m}^{-3}\right.$ ), low-temperature $\left(T_{e, \text { center }}<3 \mathrm{eV}\right)$ deuterium plasmas in the "as received" condition (i.e. unpolished and unannealed). Targets are exposed to sequential and identical $20 \mathrm{~s}$ pulses $(B=0.8 \mathrm{~T})$ to the desired fluence. Approximately $10-15 \mathrm{~min}$ between shots is required for magnet cooling. Due to strong cooling, the targets are quenched when the plasma is removed, ensuring all implanted species are "frozen" in place (i.e. diffusion is reduced by many orders of magnitude) until the next pulse is initiated. The concern is that the high ion flux from these plasmas will supersaturate the low hydrogen solubility refractory metals and create stresses in the material that are relieved through the formation of lattice defects (i.e. vacancies, dislocations, etc.). The defect sites represent trap sites for implanted hydrogen so any plasma-induced production of trap sites must be well understood to predict hydrogenic retention behaviour in future fusion devices.

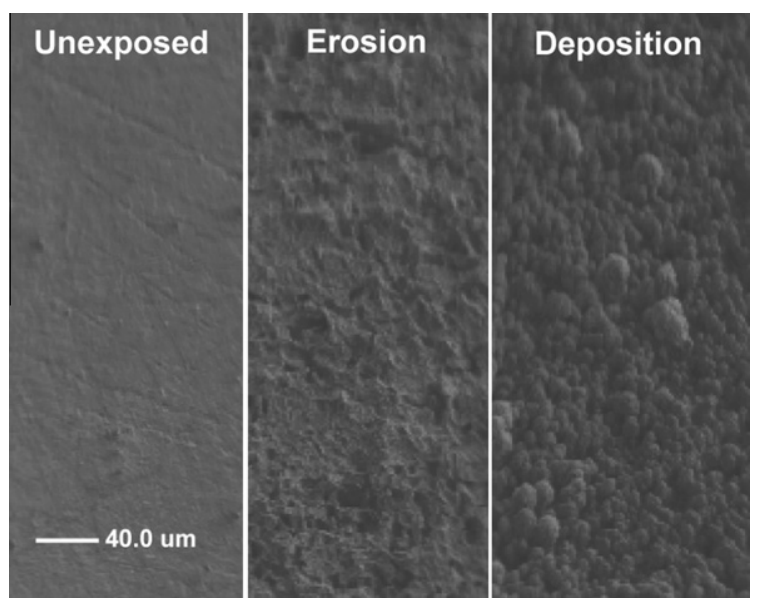

Fig. 3. SEM images of graphite surface for unexposed surface and regions of erosion and deposition. All images are on the same scale. 
Using ${ }^{3} \mathrm{He}$ NRA, local concentrations of implanted deuterium can be determined at various locations on the target surface. This spatial resolution is a great advantage since the exposed area of the targets ( $16 \mathrm{~mm}$ diameter) is of similar size as the confined plasma column ( $\sim 15 \mathrm{~mm}$ diameter). Since the plasma column has a peaked distribution of plasma flux and surface temperature (see Fig. 1) by measuring D contents at various radial locations, we examine retention properties under different plasma flux and surface temperature conditions. Using this method, Fig. 4 plots retained fraction $\left(D_{\text {retained }} / D_{\text {incident }}^{+}\right)$as a function of target surface temperature for $\mathrm{W}$ and Mo over a range of incident plasma flux densities. Plotting retained fractions normalizes the data to plasma fluence but it should be noted that previous work [8] has shown there to be no discernable fluence dependence of retention for these plasma conditions. TDS results presented in [8] for the W targets show a total retention a factor of $\sim 2$ higher than integrated NRA results, likely indicating that the retention extends beyond the $3 \mu \mathrm{m}$ probing depth of the ${ }^{3} \mathrm{He}$ ion beam. This is not surprising given the high temperatures of the exposure leading to high diffusion rates of hydrogen in $\mathrm{W}$.

Fig. 4 shows a strong temperature dependence on retention leading to exponential decay of retained fractions with increasing target surface temperature for $T_{\text {surf }}>1000 \mathrm{~K}$. For the temperature range $600-1000 \mathrm{~K}$, the retained fraction appears to be fairly constant in the order of $10^{-6}$. The different ranges of incident plasma flux density do not appear to have a strong effect on hydrogenic retention and both $\mathrm{W}$ and Mo targets display very similar retention properties. Since the measured retention levels in the "as received" tungsten targets is very low and in agreement with literature values [1618], these results indicate that extrapolating low-flux laboratory results to high flux devices is acceptable for unmodified tungsten.

However, in future fusion reactors there will be other factors that may affect hydrogenic retention and may also be modified by high plasma fluxes. Primarily, this is the presence of $14.1 \mathrm{MeV}$ neutrons from the deuterium-tritium fusion reaction. These energetic neutrons can displace lattice atoms as they pass through the material and this damage leads to creation of trap sites. In this work, heavy ions are used to create these displacements due to time and safety constraints working with neutron-irradiated materials. W targets at $300 \mathrm{~K}$ were irradiated with $12.3 \mathrm{MeV} \mathrm{W}^{4+}$ ions. Using $\mathrm{W}$ ions prevents any side effects from implantation of impurity species into the lattice, however, the damage is confined to the penetration depth of the $\mathrm{W}$ ion $\left(\sim 1.5 \mu \mathrm{m}\right.$ for $12.3 \mathrm{MeV} \mathrm{W}^{4+}$ [19], see Fig. 5a) as opposed to evenly distributed throughout the material as one would expect with neutron irradiation. This damage

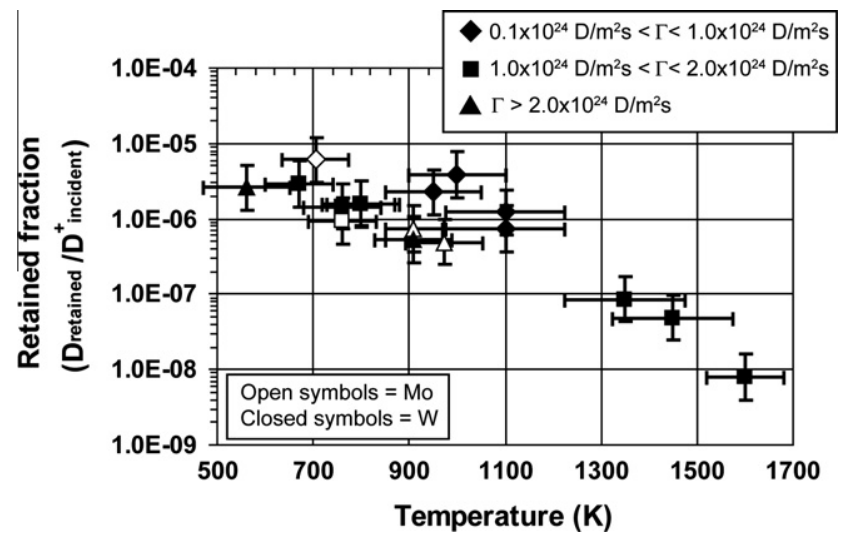

Fig. 4. Retained fractions ( $D_{\text {retained }} / D_{\text {incident }}$ ) as a function of surface temperature for $\mathrm{W}$ and Mo targets (unirradiated) exposed in Pilot-PSI. Note these data points are based on ${ }^{3} \mathrm{He}$ NRA data from different spatial locations on targets exposed for $80 \mathrm{~s}$. These different spatial locations correspond to different surface temperatures and different plasma flux densities (see Fig. 1). distribution can be smoothed by using multiple irradiation energies but we were also interested in tracking if the irradiation followed the damage profile obtained from the single irradiating energy used in this study. Peak damage levels ranging from 0.5 to 10 displacements per atom (dpa) were investigated and compared to unirradiated tungsten. The targets were then exposed to high flux deuterium plasmas in Pilot-PSI with one set of irradiated targets exposed at low surface temperatures $\left(T_{\text {surf }}<600 \mathrm{~K}\right)$ and one set exposed at high surface temperatures $\left(650 \mathrm{~K}<T_{\text {surf }}<1000 \mathrm{~K}\right.$, see Fig. 1 for example of high surface temperature profile).

The irradiation of the targets clearly leads to an enhancement of the hydrogenic retention. Both ${ }^{3} \mathrm{He}$ NRA and TDS analysis show an enhancement in retention of a factor of 2.5-4.1 compared to equivalent conditions on unirradiated $\mathrm{W}$ targets. However, there was no difference in retention for the different damage levels investigated in this work. This implies the enhancement of retention due to irradiation saturates at damage levels of $>0.5 \mathrm{dpa}$. This is very beneficial for future fusion reactors where damage levels are expected to be very high ( $20 \mathrm{dpa} /$ year $)$. The irradiation introduces a high temperature peak in the TDS spectra which dominates the total D retention (Fig. 5). High temperature desorption peaks correspond to high-energy trap sites such as voids and vacancy clusters. So it can be inferred that irradiation of the targets leads and subsequent plasma exposure leads to the formation of voids in the lattice. At lower surface temperatures, the low temperature desorption peak (corresponding to lower energy traps such as single vacancies or dislocations) is enhanced by the retention and the high temperature desorption peak is present but a factor of 2 lower than for high surface temperature plasma exposures. This may be due to the

(a)

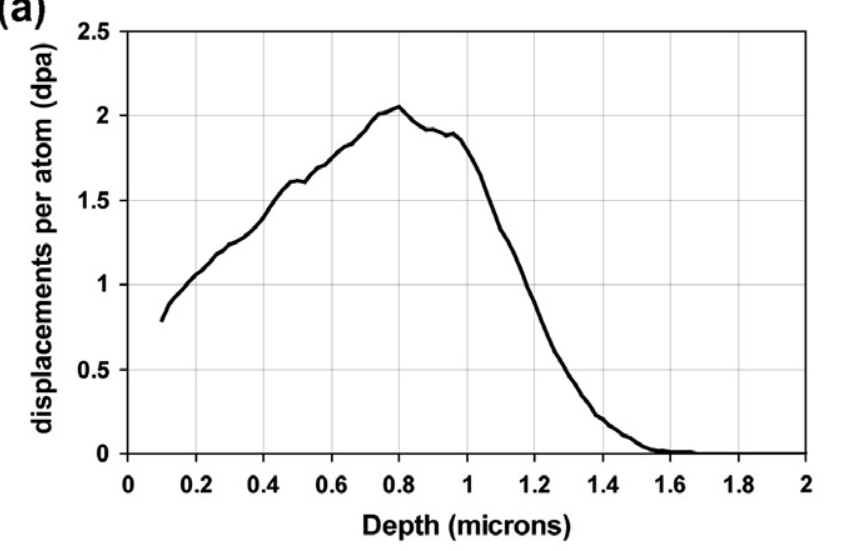

(b)

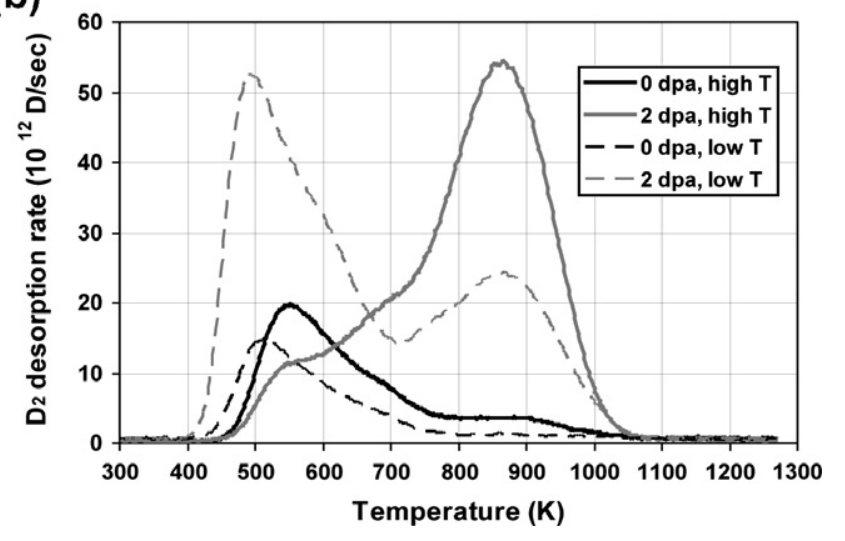

Fig. 5. (a) Damage profile from irradiation with $12.3 \mathrm{MeV} \mathrm{W}^{4+}$ ions for $2 \mathrm{dpa}$ case as determined with TRIM [17] simulations. (b) TDS spectra for W targets irradiated with $12.3 \mathrm{MeV} \mathrm{W}^{4+}$ ions to a peak damage of $2 \mathrm{dpa}$ at high $\left(T_{\text {surf }}>600 \mathrm{~K}\right)$ and low $\left(T_{\text {surf }}<600 \mathrm{~K}\right)$ surface temperatures during plasma exposure in Pilot-PSI. Unirradiated targets are also plotted for comparison. 
implanted deuterium not having full access to these irradiation traps due to restricted diffusivity at low target temperatures. Previous studies also indicate that diffusion can play an important role in retention in irradiated targets [20]. Another possible explanation is that at lower temperatures during plasma exposure, less of the damage is transformed into high-energy traps (voids) and remains in the single vacancy or dislocation state. This also would explain the enhancement of the lower temperature desorption peaks. In some high-temperature cases, desorption peaks can be seen at temperatures below the plasma exposure surface temperature. This seems contradictory, but is likely due to the thermal evolution of the target after the final plasma shot. At high exposure temperatures, some of the low-energy trap sites will remain unfilled despite a significant amount of solute $\mathrm{D}$ in the target. Once the plasma is removed, the target is quenched by the strong cooling, and the diffusion rate of the solute D drops many orders of magnitude. This makes it much more probable for the implanted D to settle into one of the unoccupied low-energy trap sites, which are accessible now that the target temperature has been reduced, rather than diffuse to a surface and recombine.

Plasma-facing materials in future fusion reactors will also experience high thermal stresses both from steady-state heatloads $\left(\leqslant 30 \mathrm{MW} / \mathrm{m}^{2}\right)$ and transient events such as ELMs $\left(\leqslant 1 \mathrm{GW} / \mathrm{m}^{2}\right)$ [21]. These thermal stresses can lead to extensive cracking of the surface. It is unclear the impact this cracking has on hydrogenic retention properties. In this investigation, $\mathrm{W}$ targets were exposed to high thermal heat loads in the JUDITH e-beam facility [22], which resulted in cracking of the surface. Two conditions were used that resulted in moderate cracking $\left(I_{\text {target }}=70 \mathrm{~mA}, \Delta t=5 \mathrm{~ms}, 100\right.$ shots $)$ and extensive cracking $\left(I_{\text {target }}=120 \mathrm{~mA}, \Delta t=5 \mathrm{~ms}, 100\right.$ shots $)$; see Fig. 6a. During the e-beam exposure the tungsten surface temperatures reach $\sim 1000 \mathrm{~K}$ for the $I_{\text {target }}=70 \mathrm{~mA}$ case and $\sim 1700 \mathrm{~K}$ for the $I_{\text {target }}=120 \mathrm{~mA}$ case. . It should be noted that the these tungsten targets had polished surfaces as opposed to other targets presented here which were exposed in the "as received" condition (i.e. rough surface). The targets with surface cracking were then exposed to high flux deuterium plasma in Pilot-PSI as well as a reference target with no prior thermal stressing in JUDITH.

TDS analysis is used to determine the retention properties of these different surface conditions. From the TDS profiles it is clear that the lower temperature desorption peaks seen on the irradiated W targets (Fig. 5) are not present meaning the lower energy traps have been removed from these targets (see Fig. 6b). This could be a result of the polishing of the surface leading to the removal of a damaged layer from the fabrication and machining processes.

Comparison of the TDS results reveals that the thermallystressed $\mathrm{W}$ has a total retention a factor of $2.1 \pm 0.2$ higher than the unstressed W. Some or perhaps all of this difference could be attributed to the slightly higher surface temperature during plasma exposure for the unstressed $\mathrm{W}\left(T_{\text {surf }} \sim 1150 \mathrm{~K}\right)$ than for the thermally-stressed $\mathrm{W}\left(T_{\text {surf }} \sim 1100 \mathrm{~K}\right)$. Although a $50 \mathrm{~K}$ difference in surface temperature may not seem like a large difference, from Fig. 4 we see this is in a region with strong temperature dependence on surface temperature. It is important to note that the difference in hydrogenic retention between the moderate cracking and extensive cracking scenarios is negligible. This indicates that any enhancement of retention is not related to the physical manifestation of the thermal stressing (i.e. cracking, surface roughening) but rather through the introduction of thermal defects in the lattice that enhance the retention.

\section{Summary}

The Pilot-PSI experiment has been used to successfully investigate plasma-surface interactions in a new high plasma flux regime with low electron temperatures. The two main focuses of this
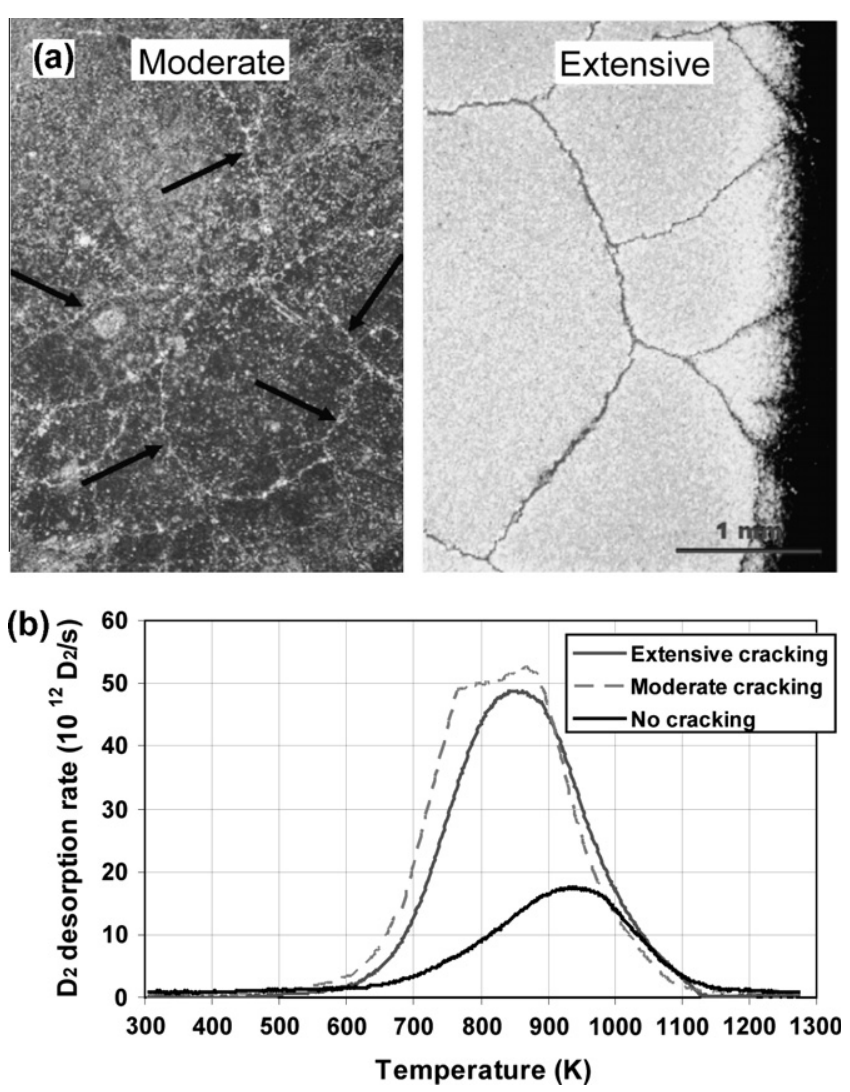

Fig. 6. (a) SEM images of $W$ targets with "moderate" and "extensive" surface cracking due to thermal cycling via. e-beam irradiation. (b) TDS spectra for thermally-stressed $\mathrm{W}$ with various degrees of surface cracking and exposed in PilotPSI. Target with no cracking was not thermally-stressed.

research program has been the chemical erosion of carbon-based materials and hydrogenic retention in tungsten and molybdenum.

Investigating fine grain graphite, carbon-fiber composites (NB31, NB41, Dunlop 3D), SiC, and diamond films (nanocrystalline and microcrystalline), the only material that had a significant difference in gross chemical erosion rate was the $\mathrm{SiC}$, which had a $\mathrm{CH}$ photon flux an order of magnitude smaller than all other carbon-based materials for $T_{e}>0.8 \mathrm{eV}$. Pyrometry and literature indicates this lower gross erosion rate of $\mathrm{SiC}$ is not a surface temperature effect. The indications from this study are that the best choice from an erosion and lifetime perspective, for a carbon-based material as a divertor plasma-facing component in future fusion devices, would be SiC.

The high-flux plasmas achievable in Pilot-PSI appear to have no significant effect on hydrogenic retention in tungsten or molybdenum. Exposing both materials over a range of fluxes, fluences, and surface temperatures, the dominant factor in determining the retention properties of the material is surface temperature. Damage induced by irradiation of $\mathrm{W}$ targets with $12.3 \mathrm{MeV} \mathrm{W}^{4+}$ ions has been found to enhance hydrogenic retention by a factor of 2.5-4.1. When exposed to plasma with high surface temperature conditions $\left(T_{\text {surf }}>650 \mathrm{~K}\right.$ ), this enhancement is due to the introduction of a high-energy trap site. However at lower surface temperatures $\left(T_{\text {surf }}<600 \mathrm{~K}\right.$ ) during plasma exposure, this enhancement is due to the addition of both high and low-energy trap sites, perhaps indicating that the defects in the material caused by the irradiation undergo further evolution during the plasma exposure, depending on the exposure conditions. Across the damage levels examined in this study (0.5-10 dpa) there was no change in retention, indicating a saturation of the enhancement of retention at levels 
$>0.5 \mathrm{dpa}$. Thermally-stressing $\mathrm{W}$ through rapid thermal cycling has also demonstrated an enhancement of hydrogenic retention by a factor of $2.1 \pm 0.2$. although a $\sim 50 \mathrm{~K}$ difference in surface temperature during plasma exposure may also be contributing to this enhancement. However, hydrogenic retention had no apparent dependence on the extent of cracking or surface roughening on the tungsten. It is likely the higher retention seen in thermallystressed $\mathrm{W}$ is due to the introduction of thermal defects which act as trap sites. This work has shown that both displacement damage and thermal cycling can enhance retention in refractory metals and that these effects must be well understood in combination with high plasma flux, in order to accurately predict hydrogenic retention behaviour in future fusion devices.

The materials research conducted on the Pilot-PSI linear plasma generator in the high plasma flux regime has yielded many new and interesting results in a new operational regime. This is encouraging for the development and impact of MAGNUM-PSI that will not only enter a new regime in plasma flux, but also in plasma fluence due to its steady-state operating capabilities. With the commissioning of MAGNUM-PSI and the continued operation of Pilot-PSI, the materials research program at FOM Rijnhuizen will be expanded in the future.

\section{Acknowledgements}

This work, supported by the European Communities under the contract of the Association EURATOM/FOM, was carried out within the framework of the European Fusion Programme with financial support from NWO. The views and opinions expressed herein do not necessarily reflect those of the European Commission.

\section{References}

[1] H.J.N. Van Eck et al., Fusion Eng. Des. 82 (2007) 1878.

[2] G.J. van Rooij, V.P. Veremiyenko, W.J. Goedheer, et al., Appl. Phys. Lett. 90 (2007) 121501.

[3] V.Kh. Alimov, K. Ertl, J. Roth, J. Nucl. Mater. 290-293 (2001) 293.

[4] M. Poon, R.G. Macaulay-Newcombe, J.W. Davis, A.A. Haasz, J. Nucl. Mater. 307311 (2002) 723

[5] O.V. Ogorodnikova, J. Roth, M. Mayer, J. Appl. Phys. 103 (2008) 034902.

[6] R.A. Anderl, D.F. Holland, et al., Fusion Technol. 21 (1992) 745.

[7] H. Eleveld, A. van Veen, J. Nucl. Mater. 212-215 (1994) 1421.

[8] G.M. Wright et al., J. Nucl. Mater. 390-391 (2009) 610.

[9] H.J. van der Meiden, R.S. Al, et al., Rev. Sci. Inst. 79 (2008) 013505.

[10] J. Westerhout, D. Borodin, et al., Phys. Scr. T138 (2009) 014017.

[11] K. Behringer, J. Nucl. Mater. 176-177 (1990) 606.

[12] B.V. Mech, A.A. Haasz, J.W. Davis, J. Nucl. Mater. 241-243 (1997) 1147

[13] M. Balden, J. Roth, J. Nucl. Mater. 280 (2000) 39.

[14] A. Kurumada, Y. Imamura, Y. Tomota, T. Oku, Y. Kubota, N. Noda, J. Nucl. Mater. 313-316 (2003) 245.

[15] M. Balden, S. Picarle, J. Roth, J. Nucl. Mater. 290-293 (2001) 47.

[16] G.N. Luo, W.M. Shu, M. Nishi, Fusion Eng. Des. 81 (2006) 957.

[17] K. Tokunaga, M.J. Baldwin, R.P. Doerner, N. Noda, et al., J. Nucl. Mater. 337-339 (2005) 887.

[18] R. Causey, K. Wilson, T. Venhaus, W. Wampler, J. Nucl. Mater. 266 (1999) 467.

[19] http://www.srim.org/index.htm.

[20] G.M. Wright, D.G. Whyte, B. Lipschultz, J. Nucl. Mater. 390-391 (2009) 544.

[21] G. Federici et al., Nucl. Fusion 41 (2001) 1967.

[22] R. Duwe, W. Kühnlein, H. Münstermann, in: Proceedings of the 18th Symposium on Fusion Technology (SOFT), Karlsruhe, Germany, October 22_l 26, 1994, p. 355. 